\title{
A Conformal Holomorphic Field Theory
}

\author{
Anatol Odzijewicz \\ Institute of Physics, Warsaw University Division in Bialystok, PL-15-424 Bialystok, \\ 41 Lipowa, Poland
}

\begin{abstract}
A formulation of a field theory on the complex Minkowski space in terms of complex differential geometry is proposed. It is also shown that our model of field theory differs from the standard model on the real Minkowski space only in the limit of high energy.
\end{abstract}

\section{Introduction}

The aim of this paper is the construction of field theory for the massive conformal particle interacting with an external field. There are important reasons, having their roots in conformal symplectic geometry to investigate such a theory on the complex Minkowski space. We believe that the utilization of the complex Minkowski space as a base for construction of the field theory is not only useful technically (see e.g. [13]), but can be of great physical importance.

In the following, by a scalar massive conformal particle we will understand a physical object localized in time and space with a given energy and momentum. The conformal particle mass may change when the particle interacts with a field, contrary to the relativistic particle mass. Due to this fact the phase space of a scalar massive conformal particle is an eight - dimensional conformal Hamiltonian space (see $[7,10])$. Considering also scalar conformal anti-particles, scalar conformal tachyons and scalar massless conformal particles we find (see Sect. 2) that two models of kinematics of conformal scalar particles exist. The phase space of the first model (which will be called the nilpotent model) is the cotangent bundle $T^{*} M$ of the conformal compactification $M$ of Minkowski space. In the second model (holomorphic one) the phase space is given as the complexification $\mathbb{M}$ of $M$. It turns out that the conformally invariant symplectic form $\omega_{h}$ on $\mathbb{M}$ depends on the real parameter $h$ and $\left(\mathbb{M}, \omega_{h}\right)$ corresponds to $\left(T^{*} M, \omega_{0}\right)$ when $h \rightarrow 0$, where $\omega_{0}$ stands for a canonical symplectic form on $T^{*} M$. In other words the nilpotent model is the limiting case of the holomorphic one.

Taking into account the above facts we construct field theory on the complex Minkowski space (the configuration space of holomorphic kinematics) which, at 
$h \rightarrow 0$, corresponds to the standard field theory on the real Minkowski space (the configuration space of the nilpotent kinematics); see Sect. 3. This formulation is carried out in terms of complex differential geometry. Thus the conformal particle states (the matter fields) can be described by holomorphic sections of a certain Hermitian bundle $\Sigma_{0} \otimes \mathbb{E}$ over a domain in $\mathbb{M}$. $\Sigma_{0}$ stands for the bundle describing spinor degrees of freedom and $\mathbb{E}$ denotes the charge bundle, i.e. the bundle connected with interaction of the conformal particle. On the other hand the gauge field is identified with the holomorphic structure of $\mathbb{E}$. The action functional for the fields is also defined and the field equations, using the variational principle, are derived in Sect. 3.

Expanding the matter fields, the gauge fields and the field equations in $h$ parameter, one can find that in zero-order approximation they correspond to analogous objects of standard field theory on real Minkowski space, see Sect. 4. Thus, in the special case, the solutions of Eqs. (4.18) generate the solutions of the Yang-Mills equations, at the limit $h \rightarrow 0$.

Many interesting problems connected with this approach are not yet solved. For example: to find solutions of (4.18) which would correspond to non-abelian solutions of the Yang-Mills equations and whether every solution of the YangMills equations is obtainable from the solution of (4.18).

\section{Possible Models of Kinematics for Conformal Scalar Massive Particle}

The possible scalar conformal kinematics and their physical interpretations are carried out in this part of the paper. The arbitrary spin case is considered elsewhere [7].

The classical conformal scalar massive particle is defined as a time-spatially localized physical object with an energy and a momentum. Just as in relativistic mechanics the conformal particles will be divided here into particles, anti-particles and tachyons. But contrary to the relativistic mechanics the mass of the particle may vary during its evolutions. Consequently the corresponding phase spaces will be the conformally homogeneous Hamiltonian symplectic manifolds of eight dimensions. The Kiryllov-Kostant-Souriau theorem says (see [1]) that each $G$-homogeneous Hamiltonian symplectic manifold covers a certain $\mathrm{Ad}^{\#}(G)$-orbit in (5*. In such a way the analysis of the massive scalar conformal particle phase space can be reduced to the analysis of 8-dimensional $\operatorname{Ad}^{\#}(\operatorname{SU}(2,2))$-orbits in $\mathrm{SU}(2,2)^{*} \cong \mathrm{SU}(2,2)$.

The following considerations require some terms of the twistor theory (see [8, $11,12])$. The conformal group $S U(2,2)$ will be treated as the automorphism group of the twistor space $\mathbb{T}$ (which is $\mathbb{C}^{4}$ with the Hermitian form $\eta$ of signature $++--)$. The conformal Lie algebra $\mathrm{SU}(2,2)$ will be realized as the set of $\mathscr{X} \in$ EndT, which satisfy $\mathscr{X}^{+} \eta+\eta \mathscr{X}=0$ and $\operatorname{Tr} \mathscr{X}=0$. The conformally compactified complex Minkowski space $\mathbb{M}$ is defined as the Grassmanian of twodimensional vector subspaces in $\mathbb{T}$. Signature of the element $z \in \mathbb{M}$ will be defined as the signature of the hermitian form $\left.\eta\right|_{z}$. The fact that $\operatorname{dim}_{\mathbb{C}} z=2$ implies that the signature of $z$ is given by a pair $k l$ where $k, l=0,+,-$. Let $\mathbb{M}^{k l}$ be the set of elements $z \in \mathbb{M}$ with signature $k l$. The submanifolds $\mathbb{M}^{k l}$ are the orbits of conformal group, which acts canonically on $\mathbb{M}$. In particular $\mathbb{M}^{00}=: M$ is the conformal compactification of the real Minkowski space. The real Minkowski 
space $M_{\infty} \hookrightarrow M$ is defined here as an affine space of elements $z \in M$ that are transversal to a certain element $\infty \in M$ which is called point in infinity. The elements $z \in M$ for which $\operatorname{dim}_{\mathbb{C}}(z \cap \infty) \geqq 1$, form the cone in infinity $C_{\infty}$. Hence $M=M_{\infty} \cup C_{\infty}$. The cone $C_{z}$, with vertex $z \in \mathbb{M}$, is defined alike, that is $z^{\prime} \in C_{z}$ iff $\operatorname{dim}_{\mathbb{C}}\left(z^{\prime} \cap z\right) \geqq 1$. The family of these cones defines the conformal structure on $\mathbb{M}$. The Poincaré group extended by dilatations is defined as stabilizer $S U(2,2)_{\infty}$ of infinity. The intersection of stabilizers $S U(2,2)_{0} \cap S U(2,2)_{\infty}$, where point $0 \in M_{\infty}$ is the origin of the Lorentz coordinates system, is the Lorentz group extended by dilatations. The Lorentz group $L_{0, \infty}$ and dilatations group $D_{0, \infty}$ are defined as commutator and centralizer of $S U(2,2)_{0} \cap S U(2,2)_{\infty}$ respectively. The group of Minkowski space translations is defined as a set of $\exp \mathscr{X}$, where $\mathscr{X} \in \mathrm{SU}(2,2)$ such that $\operatorname{Im} \mathscr{X} \subset \infty \subset \operatorname{Ker} \mathscr{X}$, while the elements $\exp \mathscr{X}$, where $\operatorname{Im} \mathscr{X} \subset 0 \subset \operatorname{Ker} \mathscr{X}$, form the group of four-accelerations.

Let us define the following three $S U(2,2)$-spaces: $\mathbb{L} \hookrightarrow M \times M$, where $(x, v) \in \mathbb{L}$ iff $x \cap v=\{0\} ; \mathbb{N} \hookrightarrow M \times S U(2,2)$, where $(x, \mathscr{X}) \in \mathbb{N}$ iff $\operatorname{Im} \mathscr{X} \subset x \subset \operatorname{Ker} \mathscr{X} ; \mathbb{I} \mathbf{M}:=\mathbb{M}^{++}$ $\cup \mathbb{M}^{+-} \cup \mathbb{M}^{--}$. The conformal group action is defined respectively as follows: $(x, v) \rightarrow(g x, g v) ;(x, \mathscr{X}) \rightarrow(g x, \operatorname{Ad}(g) \mathscr{X})$ and $z \rightarrow g z$, where $g \in S U(2,2)$. The vector bundle $\Lambda_{\mathbb{N}}: \mathbb{N} \rightarrow M$, where $\Lambda_{\mathbb{N}}$ is the projection onto the first component of the product $M \times S U(2,2)$, is of rank four and the cones $C_{x} \subset \Lambda_{\mathbb{N}}^{-1}(x)\left((x, \mathscr{X}) \in C_{x}\right.$ iff $\operatorname{dim}_{\mathbb{R}} \operatorname{Im} \mathscr{X}=1$ ) define a natural conformal structure on it. Let $\mathbb{N}$ denote $\mathbb{N}-\bigcup_{x \in M} C_{x}$.

Due to $\mathbb{T}=x \oplus v$ for $(x, v) \in \mathbb{L}$ and $\mathbb{T}=z \oplus z^{\perp}$, where $z^{\perp} \in \mathbb{I}$ is subspace of $\mathbb{T}$ orthogonal to $z$, one can define maps:

$$
\begin{gathered}
J_{\kappa}(x, v):=\kappa\left(\Pi_{x}-\Pi_{v}\right), \quad 0<\kappa \in \mathbb{R}, \\
J_{0}(x, \mathscr{X}):=\mathscr{X}, \\
J_{h} z:=i h\left(\Pi_{z}-\Pi_{z^{\perp}}\right), \quad 0 \neq h \in \mathbb{R} .
\end{gathered}
$$

$\Pi_{x}, \Pi_{v}, \Pi_{z}$, and $\Pi_{z^{\perp}}$ are projections of $\mathbb{T}$ on $x, v, z$, and $z^{\perp}$ respectively. As it is easy to check $J_{\kappa}, J_{0}$, and $J_{h}$ are conformally equivariant maps of $\mathbb{L}, \mathbb{N}$, and $\mathbb{M}$ respectively into $\mathrm{SU}(2,2)$.

Proposition 1. All of the 8-dimensional conformal Ad-orbits are given by $J_{\kappa}(\mathbb{L})$, $J_{0}(\tilde{\mathbb{N}})$, and $J_{h}(\tilde{\mathbb{M}})$, where $\kappa>0$ and $h \neq 0$, that is:

a) $\mathbb{L}$ is a conformally homogeneous space and $J_{\kappa}$ is an isomorphism of $\mathbb{L}$ on Ad $(S U(2,2))$-orbit which consists of those $\mathscr{X} \in \mathrm{SU}(2,2)$ which have $\kappa$ and $-\kappa$ as their eigenvalues.

b) $\mathbb{N}$ is the union of three $S U(2,2)$-orbits: $\mathbb{N}^{++}$the bundle of the upper halves of the interiors of cones; $\mathbb{N}^{--}$the bundle of the bottom halves of the interiors of cones; $\mathbb{N}^{-+}$the bundle of the exteriors of cones. $J_{0}$ is isomorphism of $\mathbb{N}^{++}, \mathbb{N}^{--}$, and $\mathbb{N}^{+-}$on 8-dimensional nilpotent $\operatorname{Ad}(S U(2,2))$-orbits (there are only three such orbits).

c) $\mathbb{M}^{++}, \mathbb{M}^{--}$, and $\mathbb{M}^{+-}$are isomorphically mapped by $J_{h}$ on 8-dimensional $\operatorname{Ad}(S U(2,2))$-orbits which consist of $\mathscr{X} \in \mathrm{SU}(2,2)$ with eigenvalues ih and -ih.

For the proof of proposition see [7,10].

The Kiryllov construction (see $[1,5]$ ) gives us the conformally invariant symplectic structure on the orbits $J_{k}(\mathbb{L}), J_{0}\left(\mathbb{N}^{k l}\right)$ and $J_{h}\left(\mathbb{M}^{k l}\right)$, where $k, l=+$, - . It 
can be then transported onto $\mathbb{L}, \mathbb{N}$, and $\tilde{\mathbb{M}}$ by the map $J_{\kappa}, J_{0}$, and $J_{h}$ respectively. The symplectic form obtained in this way is denoted by $\omega_{k}, \omega_{0}$, and $\omega_{h}$ respectively. Thus $\left(\mathbb{L}, \omega_{\kappa}, J_{\kappa}\right),\left(\mathbb{N}, \omega_{0}, J_{0}\right)$ and $\left(\mathbb{\mathbb { M }}, \omega_{h}, J_{h}\right)$ are the conformal Hamiltonian spaces for which $J_{k}, J_{0}$, and $J_{h}$ are the momentum maps. For the definition of the momentum map see $[1,5]$.

Proposition 2 (see [10]). $\mathbb{N}$ and $T^{*} M$ are isomorphic as vector bundles and conformal Hamiltonian spaces and the restriction of the canonical symplectic form of $T^{*} M$ to $\tilde{\mathbb{N}}$ is $\omega_{0}$.

In the symplectic geometry there exists a procedure (see e.g. [3]) which for a given phase space $(Q, \omega)$ allows as to define a configuration space $K$. This procedure is based on the notion of polarization $F \subset T^{\mathbb{C}} Q$, i.e. $\frac{1}{2} \operatorname{dim} Q$-dimensional locally integrable complex distribution, isotropic with respect to the symplectic form $\omega$. The configuration space is defined as the quotient $K:=Q / F \cap \bar{F} \cap T Q$, where $\bar{F}$ is complex conjugation of $F$. We have to assume that $F$ satisfies appropriate conditions (see [3]) which enable us to define a structure of a differential manifold on $K$. In the case of $G$-phase space we demand that $F$ be invariant with respect to the $G$-action on $Q$. Using the algebraic methods one can find that for $\left(\mathbb{L}, \omega_{\kappa}\right)$ and $\left(\mathbb{N}, \omega_{0}\right)$ the only conformally invariant polarizations are those given by projectors $\Lambda_{\mathbb{L}}:=p r_{1}: \mathbb{L} \rightarrow M$ and $\Lambda_{\mathbb{N}}:=p r_{1}: \mathbb{N} \rightarrow M$ respectively. For $\left(\mathbb{M}, \omega_{h}\right)$ the only conformally invariant polarization is given by holomorphic (anti-holomorphic) structure of $\tilde{\mathbb{M}}$. Hence $M$ is the configuration space for $\left(\mathbb{L}, \omega_{\kappa}\right)$ and $\left(\mathbb{N}, \omega_{0}\right)$, and $\tilde{\mathbb{M}}$ is the configuration space for $\left(\tilde{\mathbb{M}}, \omega_{h}\right)$.

Since $J_{0}(\tilde{\mathbb{N}})$ consists of nilpotent $\operatorname{Ad}(S U(2,2))$-orbits we shall call the conformal kinematics realized by $\left(\mathbb{N}, \omega_{0}, J_{0}\right)$ the conformal nilpotent kinematics. The kinematics realized by $\left(\mathbb{M}, \omega_{h}, J_{h}\right)$ will be called conformal holomorphic kinematics.

In order to make the physical interpretation of the above models of conformal kinematics we shall express symplectic structures, conformal group actions and momentum maps in canonical systems of coordinates. Therefore, let us take $\eta=-i\left(\begin{array}{cc}0 & -\sigma_{0} \\ \sigma_{0} & 0\end{array}\right)$, where $0=\left(\begin{array}{ll}0 & 0 \\ 0 & 0\end{array}\right)$ and $\sigma_{0}=E=\left(\begin{array}{ll}1 & 0 \\ 0 & 1\end{array}\right), \infty=\left\{\left(\begin{array}{l}\zeta \\ 0\end{array}\right): \zeta \in \mathbb{C}^{2}\right\}$ and $0=\left\{\left(\begin{array}{l}0 \\ \zeta\end{array}\right): \zeta \in \mathbb{C}^{2}\right\}$. Thus $z \in \mathbb{M}_{\infty}$ iff $z=\left\{\left(\begin{array}{c}Z \zeta \\ \zeta\end{array}\right): \zeta \in \mathbb{C}^{2}\right\}$, where $Z \in \operatorname{Mat}_{2 \times 2}(\mathbb{C})$ and $z \in M_{\infty}$ iff $Z^{+}=Z$. The above choice of $\eta, \infty$, and 0 gives us the decomposition

$$
S U(2,2)=\mathscr{T}_{\infty} \oplus \mathscr{L}_{0, \infty} \oplus \mathscr{D}_{0, \infty} \oplus \mathscr{A}_{0}
$$

into a direct sum of subalgebras, where

$$
\begin{aligned}
\mathscr{T}_{\infty} & =\left\{\left(\begin{array}{ll}
0 & T \\
0 & 0
\end{array}\right): T=T^{+} \in \operatorname{Mat}_{2 \times 2}(\mathbb{C})\right\}, \\
\mathscr{L}_{0, \infty} & =\left\{\left(\begin{array}{cc}
L & 0 \\
0 & -L^{+}
\end{array}\right): \operatorname{Tr} L=0, L \in \operatorname{Mat}_{2 \times 2}(\mathbb{C})\right\}, \\
\mathscr{D}_{0, \infty} & =\left\{d\left(\begin{array}{cc}
E & 0 \\
0 & -E
\end{array}\right): d \in \mathbb{R}\right\}, \\
\mathscr{A}_{0} & =\left\{\left(\begin{array}{ll}
0 & 0 \\
C & 0
\end{array}\right): C^{+}=C \in \operatorname{Mat}_{2 \times 2}(\mathbb{C})\right\},
\end{aligned}
$$


denote Lie subalgebras of $\mathrm{SU}(2,2)$ generating translations Lorentz transformations, dilatations and four-accelerations respectively. In our calculations $2 \times 2$ matrix coordinates are preferred. The Minkowski coordinates are given, by decompositions into Pauli matrices: $Z=z^{\mu} \sigma_{\mu}=\left(x^{\mu}+i y^{\mu}\right) \sigma_{\mu}, T=t^{\mu} \sigma_{\mu}, L=l^{0 k} \sigma_{k}$ $+\frac{1}{2} \varepsilon_{k m n} l^{k m} \sigma_{n}$ and $C=c^{\mu} \sigma_{\mu}$. These generate the basis of $\operatorname{SU}(2,2)$, consistent with (2.4):

$$
\begin{aligned}
& \mathscr{L}_{0 k}=-\mathscr{L}_{k 0}:=\left(\begin{array}{cc}
\sigma_{k} & 0 \\
0 & -\sigma_{k}
\end{array}\right), \quad \mathscr{L}_{l m}=-\mathscr{L}_{m l}:=i \varepsilon_{l m n}\left(\begin{array}{cc}
\sigma_{n} & 0 \\
0 & \sigma_{n}
\end{array}\right), \\
& \mathscr{P}_{\mu}:=\left(\begin{array}{cc}
0 & \sigma_{\mu} \\
0 & 0
\end{array}\right), \quad \mathscr{A}_{v}:=\left(\begin{array}{cc}
0 & 0 \\
\sigma_{v} & 0
\end{array}\right), \quad \mathscr{D}:=\left(\begin{array}{cc}
E & 0 \\
0 & -E
\end{array}\right) .
\end{aligned}
$$

The basis of $\operatorname{SU}(2,2)^{*} \cong \operatorname{SU}(2,2)$ dual to $(2.5)$ with respect to the Cartan-Killing form $\left\langle\mathscr{X}, \mathscr{X}^{\prime}\right\rangle:=\frac{1}{2} \operatorname{Tr} \mathscr{X} \mathscr{X}^{\prime}$ is

$$
\mathscr{L}_{k l}^{*}=\frac{1}{2} \mathscr{L}_{k l}, \mathscr{L}_{0 k}=\frac{1}{2} \mathscr{L}_{0 k}, \mathscr{P}_{\mu}^{*}=\mathscr{A}_{\mu}, \mathscr{A}_{v}^{*}=\mathscr{P}_{v}, \mathscr{D}^{*}=\frac{1}{2} \mathscr{D} .
$$

Expressing the momentum map $J: Q \rightarrow S U(2,2)$ in terms of the dual basis,

$$
J(q)=\frac{1}{2} m^{\mu \nu}(q) \mathscr{L}_{\mu \nu}^{*}+p^{\mu}(q) \mathscr{P}_{\mu}^{*}+a^{v}(q) \mathscr{A}_{v}^{*}+d(q) \mathscr{D}^{*}
$$

one can find: 4-momentum $p^{\mu}(q)$, angular momentum $m^{\mu v}(q)$, 4-acceleration $a^{v}(q)$ and dilatation $d(q)$ for $q \in Q$. Introducing the matrix coordinates $P=p^{\mu} \sigma_{\mu}$, $M=\frac{1}{2} m^{0 k} \sigma_{k}-\frac{i}{4} m^{k l} \varepsilon_{k l n} \sigma_{n}$ and $A=a^{v} \sigma_{v}$, we obtain

$$
J(q)=\left[\begin{array}{c}
\frac{d(q)}{2}+M(q), A(q) \\
P(q),-\frac{d(q)}{2}-M^{+}(q)
\end{array}\right] .
$$

One can also parametrize elements of $Q(Q=\mathbb{L}, \mathbb{N}, \mathbb{M})$ by matrix coordinates. The simple considerations show that for the specified phase spaces we have:

1) the fibre element $v \in \Lambda^{-1}(x)$, where $x \in M_{\infty}$ is of the form $v=\left\{\left[\begin{array}{c}(E+X V) \zeta \\ V \zeta\end{array}\right]: \zeta \in \mathbb{C}^{2}\right\}$. Hence, the pairs of matrices $(X, V) \in H(2) \times H(2)$, where $H(2)$ denotes vector space $2 \times 2$ Hermitian matrices, define the system of coordinates on $\Lambda_{\mathbb{L}}^{-1}\left(M_{\infty}\right)$.

2) $\mathscr{X} \in \Lambda_{\mathbb{N}}^{-1}(x)$ iff $\mathscr{X}=\left[\begin{array}{cc}X S, & -X S X \\ S, & -S X\end{array}\right]$, where $(X, S) \in H(2) \times H(2)$.

3) elements $z \in \mathbb{M}_{\infty}$ are parametrized by pairs of Hermitian conjugated matrices $\left(Z, Z^{+}\right), Z \in \operatorname{Mat}_{2 \times 2}(\mathbb{C})$. After some calculations we obtain

$$
\begin{gathered}
J_{\kappa}(q)=\left[\begin{array}{cc}
-E-2 X V, & 2 X(E+V X) \\
-2 V, & E+2 V X
\end{array}\right] \text { for } q=(x, v) \in \Lambda_{\mathbb{L}}^{-1}\left(M_{\infty}\right), \\
J_{0}(q)=\left[\begin{array}{cc}
X S, & -X S X \\
S, & -S X
\end{array}\right] \text { for } q=(x, \mathscr{X}) \in \Lambda_{\mathbb{N}}^{-1}\left(M_{\infty}\right), \\
J_{h}(q)=i h\left[\begin{array}{c}
\left(Z+Z^{+}\right)\left(Z-Z^{+}\right)^{-1},-2 Z\left(Z-Z^{+}\right)^{-1} Z^{+} \\
2\left(Z-Z^{+}\right)^{-1},-E-2\left(Z-Z^{+}\right)^{-1} Z^{+}
\end{array}\right] \text {for } z \in \mathbb{M}_{\infty} .
\end{gathered}
$$


Comparing (2.9), (2.10), and (2.11) with (2.8) we get $P=-2 \kappa V, P=S$ and $P=h\left(\frac{Z-Z^{+}}{2 i}\right)^{-1}$ respectively. Hence, $(X, P) \in H(2) \times H(2)$ may be used as canonical coordinates common for the three cases.

Symplectic forms $\omega_{\kappa}, \omega_{0}$, and $\omega_{h}$ expressed in $X=x^{\mu} \sigma_{\mu}, P=p^{\mu} \sigma_{\mu}$ as one should expect, have the canonical form

$$
\omega=\operatorname{Tr}(d X \wedge d P)=d x^{\mu} \wedge d p_{\mu} .
$$

Expressing in $(X, P)$ the Poincare group action and relativistic kinematical quantities connected with it we find that they are the same for each of the considered models of kinematics. The differences among the models appear if we go out of the relativistic mechanics, i.e. if we also consider the four-accelerations and dilatations.

$$
\begin{array}{r}
d=\frac{1}{2} \operatorname{Tr} X P-\kappa, \quad A=-X P X-2 \kappa X \text { for } \mathbb{L}, \\
\quad d=\frac{1}{2} \operatorname{Tr} X P, \quad A=-X P X \text { for } \mathbb{N}, \\
d=\frac{1}{2} \operatorname{Tr} X P, \quad A=-X P X-h^{2} P^{-1} \text { for } \mathbb{M} .
\end{array}
$$

For the acceleration transformations $\left(\begin{array}{ll}E & 0 \\ C & E\end{array}\right) \in S U(2,2)$, where $C \in H(2)$, we have respectively

$$
\begin{gathered}
X^{\prime}=X(C X+E)^{-1}, \quad P^{\prime}=(C X+E) P(X C+E)-2 \kappa C(X C+E), \\
X^{\prime}=X(C X+E)^{-1}, \quad P^{\prime}=(C X+E) P(X C+E), \\
X^{\prime}=\left[X P+i h E-i h\left(X C-i h P^{-1} C+E\right)\right]^{-1}(C X P+i h C+P)^{-1} \\
P^{\prime}=(C X+E) P(X C+E)+h^{2} C P^{-1} C .
\end{gathered}
$$

From (2.13-18) we can see that the models $\mathbb{L}$ and $\mathbb{M}$ correspond to the nilpotent model when $\kappa \rightarrow 0$ and $h \rightarrow 0$ respectively. Thus nilpotent conformal kinematics is, in some sense, the limit case of the two others. The differences among kinematics are significant for big parameters $\kappa$ and $h$.

On the contrary to $\mathbb{N}=\mathbb{N}^{++} \cup \mathbb{N}^{+-} \cup \mathbb{N}^{--}$and $\tilde{M}=\mathbb{M}^{++} \cup \mathbb{M}^{+-} \cup \mathbb{M}^{--}$ the phase space $\mathbb{L}$ is conformally homogeneous. An element $q \in \mathbb{N}(\tilde{\mathbb{M}})$ with the canonical coordinates $\left(x^{\mu}, p^{v}\right)$ belongs to $\mathbb{N}^{++}\left(\mathbb{M}^{++}\right)$if $p^{0}>0$ and $p^{02}-p^{2}>0$, $q \in \mathbb{N}^{--}\left(\mathbb{M}^{--}\right)$if $p^{0}<0$ and $p^{02}-\mathbf{p}^{2}>0$, while $q \in \mathbb{N}^{+-}\left(\mathbb{M}^{+-}\right)$if $p^{02}-\mathbf{p}^{2}<0$. In such a way the conformal group orbits $\mathbb{M}^{++}, \mathbb{M}^{--}$, and $\mathbb{M}^{+-}\left(\mathbb{N}^{++}, \mathbb{N}^{--}\right.$, and $\mathbb{N}^{+-}$) form the phase spaces for conformal scalar massive particle, anti-particle and conformal scalar tachyon. Concluding, we state that holomorphic conformal kinematics can be used for the description of conformal massive particle (antiparticle and tachyon) equally well as the nilpotent one.

Finally let us assume that $h$ is the Planck constant (because of $P=h Y^{-1} h$ has the dimension of the action). Thus we get $y^{\mu}=\frac{h}{m c} \frac{p^{\mu}}{m c}$, where $\frac{h}{m c}$ is the length of the Compton wave and $m c=\sqrt{p^{02}-\mathbf{p}^{2}}$ is the relativistic mass of particle. One can see that the neighbourhood $\Omega$ of $M$ in $\mathbb{M}$ consists of massive $\left(\right.$ small $\left.\frac{h}{m c}\right)$ conformal 
particles moving with small velocity $\left(\operatorname{small} \frac{p^{\mu}}{m c}\right)$

Fig. 1

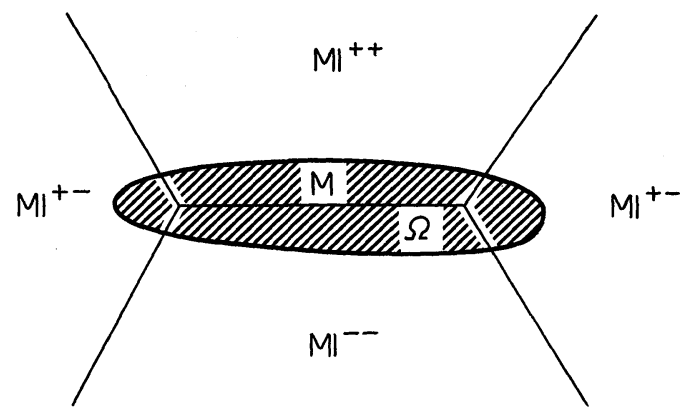

The analysis of transformation rules (2.16), (2.18) and expressions for dilatation and 4-acceleration (2.13), (2.15) leads us to the conclusion that the difference between the holomorphic and the nilpotent conformal kinematics becomes significant only when we go beyond $\Omega$, that is when particles of a small mass $\left(\right.$ big $\left.\frac{h}{m c}\right)$ and large velocity $\left(\frac{p^{\mu}}{m c} \gg 1\right)$ are taken into account. Thus the answer to the question whether the nilpotent or the holomorphic model is realisable in nature has to be sought in high energy physics.

\section{The Field Theory on $\mathbb{M}^{++} \cup \mathbb{M}^{--}$}

In this section we present a field theory for the massive conformal particle which will be consistent with the conformal holomorphic kinematics. All the constructions presented below will be made in terms of complex geometry of $\mathbb{M}^{++} \cup \mathbb{M}^{--}$, i.e. of the configuration space of massive conformal scalar particle and antiparticle.

By definition the field describing the conformal massive particle is given as the holomorphic section $\psi \in H^{0}\left(\mathbb{M}^{++} \cup \mathbb{M}^{--}, \mathcal{O}(\Sigma)\right)$ of some Hermitian vector bundle $\Sigma \rightarrow \mathbb{M}^{++} \cup \mathbb{M}^{--}$, i.e. holomorphic bundle with Hermitian metric (see [4]). Analogously to field theory on the real time-space we assume that $\Sigma$ is the tensor product $\Sigma=\Sigma_{0} \otimes \mathbb{E}$ of Hermitian vector bundles $\Sigma_{0}$ and $\mathbb{E}$ which describe spinor and charge degrees of freedom of the conformal massive particle.

In view of the twistor description of $\mathbb{M}$ there is a canonical construction of spinor bundle $\Sigma_{0}$ (see [8]). To see this, let us consider holomorphic vector bundles $\mathbb{S} \rightarrow \mathbb{M}, \mathbb{S}^{\perp} \rightarrow \mathbb{M}$, and $\tilde{\mathbb{T}}$, where $\mathbb{S}_{z}=z, \mathbb{S}_{z}^{\perp}=z^{\perp}$ and $\tilde{\mathbb{T}}=\mathbb{M} \times \mathbb{T}$. Restricting the above bundles to $\mathbb{M}^{++} \cup \mathbb{M}^{--}$we obtain the holomorphic vector bundles isomorphism $\tilde{\mathbb{T}} / \mathbb{S}=\mathbb{S}^{\perp}$, which is induced by the twistor form $\eta$. The twistor form also define the Hermitian metric on $\boldsymbol{S}$ and $\boldsymbol{S}^{\perp}$. Hence, $\boldsymbol{\Sigma}_{0}$ is the Hermitian vector bundle as an algebraic combination of the Hermitian vector bundles $\mathbf{S}, \mathbf{S}^{*}$, $\tilde{\mathbb{T}} / \mathbf{S}=\boldsymbol{S}^{\perp}$ and $\left(\mathbf{S}^{\perp}\right)^{*}$. Note that by $T_{\mathbb{M}}^{*(1,0)} \cong \mathbf{S} \otimes \tilde{\mathbb{T}} / \mathbf{S}$ and $T_{\mathbb{M}}^{(1,0)} \cong \mathbb{S}^{*} \otimes(\tilde{\mathbb{T}} / \mathbf{S})^{*}$ (see [8]) any tensor bundle over $\mathbb{M}^{++} \cup \mathbb{M}^{--}$is also canonically Hermitian.

The Laplace transform enables us to study the field $\psi_{+}:=\left.\psi\right|_{\mathbb{M}^{++}}$and $\psi_{-}:=\left.\psi\right|_{\mathbb{M}^{-}}$in terms of the tempered distribution on the future and the past 
cones (see [6]). For this reason one can interprete $\psi_{+}$as matter field (positive frequency) and $\psi_{\text {- }}$ as anti-matter field (negative frequency).

There is a natural anti-isomorphism $\mathscr{C}$ (the "charge conjugation") between vector space of matter fields and vector space of anti-matter field. In order to define it let us consider the following commutative diagrams

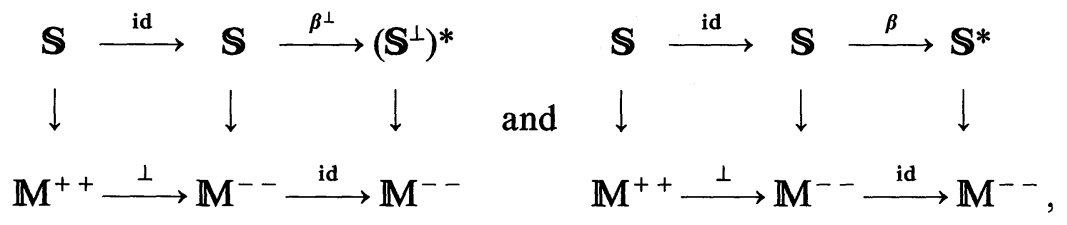

where $\beta$ and $\beta^{\perp}$ are vector bundle anti-isomorphisms defined by hermitian metric of the respective bundle and involution $\perp: \mathbb{M} \rightarrow \mathbb{M}$ maps $z \in \mathbb{M}$ on its orthogonal complement in the twistor space. One can see from the diagrams that $\left(C \psi_{+}\right)(z):=\beta^{\perp} \psi_{+}\left(z^{\perp}\right)$ and $\left(C^{\perp} \psi_{+}\right)(z):=\beta \psi_{+}\left(z^{\perp}\right)$ define anti-isomorphisms $C: H^{0}\left(\mathbb{M}^{++}, \mathcal{O}(\mathbb{S})\right) \rightarrow H^{0}\left(\mathbb{M}^{--}, \mathcal{O}\left(\mathbb{S}^{\perp *}\right)\right) \quad$ and $\quad C^{\perp}: H^{0}\left(\mathbb{M}^{++}, \mathcal{O}\left(\mathbb{S}^{\perp}\right)\right)$ $\rightarrow H^{0}\left(\mathbb{M}^{--}, \mathcal{O}\left(\mathbb{S}^{*}\right)\right)$ respectively. The anti-isomorphism $\mathscr{C}: H^{0}\left(\mathbb{M}^{++}, \mathcal{O}\left(\Sigma_{0}\right)\right)$ $\rightarrow H^{0}\left(\mathbb{M}^{--}, \mathcal{O}\left(\tilde{\Sigma}_{0}\right)\right)$ is defined accordingly to spinor structure of $\Sigma_{0}$ as an algebraic combination of $C$ and $C^{\perp}$. The Hermitian vector bundle $\tilde{\Sigma}_{0}$ is obtained from $\Sigma_{0}$ by the exchange of $\mathbb{S}$ and $\mathbb{S}^{\perp}$ for $\left(\boldsymbol{S}^{\perp}\right)^{*}$ and $\boldsymbol{S}^{*}$ respectively.

Finally, let us note that the above construction of $\Sigma_{0}$ admits a unitary representation of the conformal group in $H^{0}\left(\mathbb{M}^{++} \cup \mathbb{M}^{--}, \mathcal{O}\left(\Sigma_{0}\right)\right.$ ) (see [6]). For the charge bundle $\mathbb{E} \rightarrow \mathbb{M}^{++} \cup \mathbb{M}^{--}$, on the contrary to the case of the spinor bundle, there is not canonically defined Hermitian vector bundle structure. Therefore the basic idea of this section is to fix a Hermitian metric $H$ on $\mathbb{E}$ and interpret the possible holomorphic structure of $\mathbb{E}$ as the fields responsible for the interaction of the conformal particles (anti-particles), i.e. as the gauge field. The rank of $\mathbb{E}$ will depend on the type of interaction.

Let us now recall some facts concerning the notion of the holomorphic vector bundle. One of the ways to give the holomorphic structure on the smooth vector bundle is to introduce the Cauchy-Riemann operator $\bar{D}: C^{\infty}(\Omega, \mathbb{E})$ $\rightarrow C^{\infty}\left(\Omega, \mathbb{E} \otimes T^{*(0,1)}\right), \Omega \subset \mathbb{M}^{++} \cup \mathbb{M}^{--}$, which by definition satisfies
a) $\bar{D}^{2}=0$,
b) $\bar{D}(h \psi)=\bar{\partial} h \otimes \psi+h \bar{D} \psi$

where $h \in C^{\infty}(\Omega)$ and $\psi \in C^{\infty}(\Omega, \mathbb{E})$ (see [2]). The holomorphic sections $\psi \in H^{0}(\Omega, \mathcal{O}(\mathbb{E}))$ are then defined by the condition

$$
\bar{D} \psi=0 \text {. }
$$

Let $s_{1}, \ldots, s_{n}, n=\mathrm{rk} \mathbb{E}$, be a holomorphic frame, i.e. $\bar{D} s_{i}=0$. Because $\mathbb{M}^{++} \cup \mathbb{M}^{--}$ is topologically trivial one can define $s_{i}$ globally, $s_{i} \in H^{0}\left(\mathbb{M}^{++} \cup \mathbb{M}^{--}, \mathcal{O}(\mathbb{E})\right)$. Thus $\psi=\psi^{i} s_{i}$ is holomorphic iff $\psi^{i} \in \mathcal{O}\left(\mathbb{M}^{++} \cup \mathbb{M}^{--}\right)$. In unitary frame $u_{1}, \ldots, u_{n} \in C^{\infty}\left(\mathbb{M}^{++} \cup \mathbb{M}^{--}, \mathbb{E}\right), \quad H\left(u_{i}, u_{j}\right)=\delta_{i j}$, we have $\bar{D} u_{i}=c_{i}^{k} \otimes u_{k}$, where $c=\left[c_{i}^{k}\right] \in C^{\infty}\left(\mathbb{M}^{++} \cup \mathbb{M}^{--}, \operatorname{End}\left(\mathbb{C}^{*}\right) \otimes T^{*(0,1)}\left(\mathbb{M}^{++} \cup \mathbb{M}^{--}\right)\right)$. The condition $\bar{D}^{2}=0$ implies

$$
\overline{\partial c}-c \wedge c=0
$$


Acting with $\bar{D}$ on $s_{i}=f_{i}^{k} u_{k}$, where $f=\left[f_{i}^{k}\right] \in C^{\infty}\left(\mathbb{M}^{++} \cup \mathbb{M}^{--}, \operatorname{GL}(n, \mathbb{C})\right)$ is transition function from unitary to holomorphic frame, we get $c=-f^{-1} \bar{\partial} f$, which is a solution of (3.3). Since, the Hermitian structure of $\mathbb{E}$ was fixed the transition function $f$ defines the holomorphic structure of it. Everywhere below, where it is necessary, $f$ or $c$ is used for the description of the holomorphic structure of the bundle.

In the following considerations the metric connection $\nabla$ is used. It is unambiguously defined by the consistency conditions:

a) $\nabla^{(0,1)}=\bar{\partial}$,

b) $d H(\psi, \varphi)=H(\nabla \psi, \varphi)+H(\psi, \nabla \varphi)$,

where $\psi, \varphi \in C^{\infty}\left(\mathbb{M}^{++} \cup \mathbb{M}^{--}, \mathbb{E}\right)$, with holomorphic and Hermitian structure of $\mathbb{E}$ (see [4]).

Let $\theta$ and $\Theta=\operatorname{curv} \nabla$ mean the connection form and the curvature form of $\nabla$, respectively. Thus we have

$$
\begin{gathered}
H^{s}=f^{+} f, \\
\bar{D}^{s}=\bar{\partial}, \\
\theta^{s}=H^{s^{-1}} \partial H^{s}, \\
\Theta^{s}=\bar{\partial} \theta^{s}=: \Theta_{\mu \bar{\nu}}^{s} d z^{\mu} \wedge d \bar{z}^{\nu},
\end{gathered}
$$

where index $s$ means that a indexed quantity is written in holomorphic frame $\left\{s_{i}\right\}$. In the case of unitary frame we have

$$
\begin{gathered}
H^{u}=\mathrm{id}, \\
\bar{D}^{u}=\bar{\partial}+c, \\
\theta^{u}=-f^{-1} \bar{\partial} f+\left(\partial f^{+}\right) f^{+-1}, \\
\Theta^{u}=f^{-1} \Theta^{s} f, \quad \Theta^{u^{+}}=-\Theta^{u} .
\end{gathered}
$$

For the further considerations we shall also need

$$
\begin{gathered}
\theta^{u}=c-c^{+}=c_{\bar{v}} d \bar{z}^{\nu}-c_{\mu}^{+} d z^{\mu} \\
\Theta^{u}=\partial c-\bar{\partial} c^{+}+c \wedge c^{+}+c^{+} \wedge c=\left(\frac{\partial c_{\bar{v}}}{\partial z^{\mu}}+\frac{\partial c_{\mu}^{+}}{\partial \bar{z}^{\nu}}+\left[c_{\mu}^{+}, c_{\bar{v}}\right]\right) d z^{\mu} \wedge d \bar{z}^{\nu} .
\end{gathered}
$$

With each type of frame, respective gauge transformations are linked. Namely, let $\left\{s_{i}^{\prime}\right\}$ and $\left\{u_{i}^{\prime}\right\}$ be new holomorphic and unitary frames. Then $s_{i}^{\prime}=g_{i}^{k} s_{k}$ and $u_{i}^{\prime}=u_{i}^{k} u_{k}$, where $g=\left[g_{i}^{k}\right] \in \mathcal{O}\left(\mathbb{M}^{++} \cup \mathbb{M}^{--}, \operatorname{GL}(n, \mathbb{C})\right)$ and $u=\left[u_{i}^{k}\right] \in C^{\infty}\left(\mathbb{M}^{++}\right.$ $\left.\cup \mathbb{M}^{--}, U(n)\right)$. The new transition map $f^{\prime}=\left[f_{i}^{\prime k}\right], s_{i}^{\prime}=f_{i}^{\prime k} u_{k}^{\prime}$ is related to the former one by

$$
f^{\prime}=g f u^{-1} .
$$

The gauge transformations for the quantities given by (3.4-11) can be easily calculated from (3.14), since they are expressed by the transition map $f$.

Now let us define the action functional $S=S[\psi, c]$ for the matter $\psi$ and gauge $c$ fields. For the simplicity of calculations we shall restrict our considerations to 
scalar case, i.e. $r k \Sigma_{0}=1$. Then, by definition,

$$
\begin{aligned}
\mathscr{L}\left(c, c^{+}, \psi^{u^{+}}, \psi^{u}\right)= & \operatorname{Tr} \Theta^{u} \wedge * \Theta^{u}+\left[(\bar{\partial}+c) \psi^{u}\right]^{+} \wedge *\left(\partial-c^{+}\right) \psi^{u} \\
& +\left[\left(\partial-c^{+}\right) \psi^{u}\right]^{+} \wedge *(\bar{\partial}+c) \psi^{u}-V\left(\psi^{u^{+}} \psi^{u}\right) d z^{0} \wedge \ldots \wedge d \bar{z}^{3}
\end{aligned}
$$

will be the Lagrangian density expressed in the unitary frame.

$$
* \omega:=\omega_{\alpha_{1} \ldots \alpha_{k} \bar{\beta}_{1} \ldots \bar{\beta}_{l}} *\left(d z^{\alpha_{1}} \wedge \ldots \wedge d z^{\alpha_{k}}\right) \wedge *\left(d \bar{z}^{\bar{\beta}_{1}} \wedge \ldots \wedge d \bar{z}^{\bar{\beta}_{1}}\right)
$$

is dual of $\omega=\omega_{\alpha_{1} \ldots \alpha_{k} \bar{\beta}_{1} \ldots \bar{\beta}_{l}}, d z^{\alpha_{1}} \wedge \ldots \wedge d z^{\alpha_{k}} \wedge d \bar{z}^{\bar{\beta}_{1}} \wedge \ldots \wedge d \bar{z}^{\bar{\beta}_{1}} \in C^{\infty}\left(\mathbb{M}^{++} \cup \mathbb{M}^{--}\right.$, $\left.\mathbb{E} \otimes T^{*(k, l)}\right)$, where $*$ is the star operator given by the Minkowski metric on $\mathbb{M}_{\infty}$ $\supset \mathbb{M}^{++} \cup \mathbb{M}^{--}$. The term $V\left(\psi^{u^{+}} \psi^{u}\right)$, where $V$ is real valued function, describes selfinteraction potential of the matter field. The physical sense of the other terms in (3.15) is determined by their form. It is easy to show that (3.15) is relativistic and unitary gauge invariant.

Let us emphasize that in variational principle $c, c^{+}, \psi^{u}, \psi^{u^{+}}$will appear as independent quantities. Thus, we must include the terms related to constraints (3.2) and (3.3) in the action functional. We have

$$
\begin{aligned}
& S\left[c, c^{+}, \psi^{u}, \psi^{u^{+}}, \lambda, \Lambda\right]=\int_{\mathbb{M}^{++} \cup \mathbb{M}^{-}} \mathscr{L}\left(c, c^{+}, \psi^{u}, \psi^{u^{+}}\right)+\left[(\bar{\partial}+c) \psi^{u}\right]^{+} \wedge * \lambda \\
& +\lambda^{+} \wedge *(\bar{\partial}+c) \psi^{u}+\operatorname{Tr}[(\bar{\partial} c-c \wedge c) \wedge * \Lambda]+\operatorname{Tr}\left[\Lambda^{+} \wedge *\left(\partial c^{+}-c^{+} \wedge c^{+}\right)\right],
\end{aligned}
$$

where $\lambda \in C^{\infty}\left(\mathbb{E} \otimes T^{*(1,0)}\right)$ and $\Lambda \in C^{\infty}\left(E n d \mathbb{E} \otimes T^{*(0,2)}\right)$ are the appropriate Lagrangian factors. Assuming the transformation rules $\lambda^{\prime}=u^{-1} \lambda$ and $\Lambda^{\prime}=u^{-1} \Lambda u$ for the Lagrangian factors we guarantee unitary gauge invariance of (3.16).

The variational principle applied to (3.16) gives the following field equations

$$
\begin{aligned}
2[ & \left.* \partial * \Theta^{u}+*\left(* \Theta^{u} \wedge c^{+}\right)-*\left(c^{+} \wedge * \Theta^{u}\right)\right]-\left[(\bar{\partial}+c) \psi^{u}\right] \psi^{u^{+}} \\
& +\psi^{u}\left[\left(\partial-c^{+}\right) \psi^{u}\right]^{+}+\psi^{u} \lambda^{+}+* \bar{\partial} * \Lambda \\
& +*(c \wedge * \Lambda)-*(* \Lambda \wedge c)=0, \\
*\left(\partial-c^{+}\right) * & \left(\partial-c^{+}\right) \psi^{u}+*(\bar{\partial}+c) *(\bar{\partial}+c) \psi^{u}+V^{\prime}\left(\psi^{u^{+}} \psi^{u}\right) \psi^{u}+*\left(\partial-c^{+}\right) * \lambda=0
\end{aligned}
$$

and their hermitian conjugated counterparts. Because of its technical character the calculation of (3.17) and (3.18) is omitted here. Let us note only that we assumed that $\delta c, \delta c^{+}, \delta \psi^{u}, \delta \psi^{u^{+}}=0$ on $\partial\left(\mathbb{M}^{++} \cup \mathbb{M}^{--}\right)$and used Stokes theorem as it always holds in such cases. The variation of (3.16) with respect of $\lambda$ and $\Lambda$ gives (3.2) and (3.3) respectively.

At the end of this section let us rewrite (3.17), (3.18) and their Hermitian conjugated counterparts in the holomorphic frame. Using (3.2), (3.3) and formulae (3.4-11) we find

$$
\left.\begin{array}{rl}
* \partial *\left(H \Theta^{s} H^{-1}\right) & +H \psi^{s}\left(\nabla \psi^{s}\right)^{+}+H \psi^{s}\left(f^{-1} \lambda\right)^{+}+H\left(* \bar{\partial} * f^{-1} \Lambda f\right) H^{-1}=0 \\
-* \bar{\partial} * \Theta^{s}+ & \left(\nabla \psi^{s}\right) \psi^{s^{+}} H+f^{-1} \lambda \psi^{s^{+}} H+H^{-1}\left(* \partial *\left(f^{-1} \Lambda f\right)^{+}\right) H=0, \\
& * \nabla * \nabla \psi^{s}+V^{\prime}\left(\psi^{s^{+}} H \psi^{s}\right) \psi^{s}+* \nabla * f^{-1} \lambda=0, \\
* & \bar{\partial} *\left(\nabla \psi^{s}\right)^{+}-*\left(\nabla \psi^{s}\right)^{+} \wedge(\bar{\partial} H) H^{-1}+V^{\prime}\left(\psi^{s^{+}} H \psi^{s}\right) \psi^{s^{+}} \\
+ & * \bar{\partial} *\left(f^{-1} \lambda\right)^{+}-*\left(*\left(f^{-1} \lambda\right)^{+} \wedge(\bar{\partial} H) H^{-1}\right)=0,
\end{array}\right\}
$$


respectively. The field Eqs. (3.17) and (3.18) [(3.19) and (3.20)] are gauge equivariant in the sense of gauge transformation (3.14). It is a consequence of the gauge invariance of the action functional (3.16).

Let us also note that all quantities appearing in (3.16) are equivariant with respect to biholomorphic transformations of $\mathbb{M}^{++} \cup \mathbb{M}^{--}$. Therefore, the above considerations can be repeated in the case of general complex manifolds.

\section{Correspondence to Yang-Mills Theory}

We shall study here the behaviour of the conformal holomorphic field theory in the case when the parameter $h$ is small $(h \approx 0)$. It was shown in Sect. 2 , that the holomorphic conformal kinematics corresponds to the nilpotent conformal kinematics, i.e. standard one, which suggests the analogous correspondence on the field theory level. In order to establish it we will express all interesting as quantities and equations in canonical coordinates $\left(x^{\mu}, p^{\nu}\right)$ and next expand them in powers of $h$. We will also prefer the holomorphic frame, which is more convenient for our calculations.

Because of $z^{\mu}=x^{\mu}+i y^{\mu}=x^{\mu}+i h \frac{p^{\mu}}{p^{2}}$ the condition $h \approx 0$ implies $y \approx 0$. Everywhere below we assume that $\mathbb{E} \rightarrow \mathbb{M}^{++} \cup \mathbb{M}^{--}$and thus frames $s_{i}^{+}=\left.s_{i}\right|_{\mathbb{M}^{++}}$, $s_{i}^{-}=\left.s_{i}\right|_{\mathbb{M}^{--}}, u_{i}^{+}=\left.u_{i}\right|_{\mathbb{M}^{+}}$and $u_{i}^{-}=\left.u_{i}\right|_{\mathbb{M}^{--}}, i=1, \ldots, n$, extends in a smooth way to $M_{\infty}$ and that

$$
\begin{gathered}
\lim _{y \rightarrow+0} s_{i}^{+}=\lim _{y \rightarrow-0} s_{i}^{-}=: s_{i}^{0}, \\
\lim _{y \rightarrow+0} u_{i}^{+}=\lim _{y \rightarrow-0} u_{i}^{-}=: u_{i}^{0}, \\
s_{i}^{0}=u_{i}^{0},
\end{gathered}
$$

where $\left\{s_{i}^{0}\right\},\left\{u_{i}^{0}\right\}$ are frames of the boundary vector bundle $E \rightarrow M_{\infty}$. The conditions (4.1) and (4.2) allow us to compare the boundary values $\psi_{+}(x)=\left(\lim _{y \rightarrow+0} \psi_{+}^{i}(z)\right) s_{i}^{0}(x)$ and $\psi_{-}(x)=\left(\lim _{y \rightarrow+0} \psi_{-}^{i}(z)\right) s_{i}^{0}(x)$ of positive $\psi_{+}(z)$ $=\psi_{+}^{i}(z) s_{i}^{+}(z)$ and negative $\psi_{-}(z)=\psi_{-}^{i}(z) s_{i}^{-}(z)$ frequency matter fields. The condition (4.3) has technical character and does not restrict the generality of the discussed problem. In general the field $\psi(x)=\psi_{+}(x)+\psi_{-}(x)$ is a hyperfunction on $M_{\infty}$ (see [14]).

The gauge field in our model is expressed by transition map $f: \mathbb{M}^{++} \cup \mathbb{M}^{--}$ $\rightarrow \mathrm{GL}(n, \mathbb{C})$. Because of Gauss decomposition $f=R U$, where $R^{+}=R$ and $U^{+}=U^{-1}$, one can choose a unitary frame in such a way that $f=R=\exp A$, where $A=A^{+}$. Thus, the gauge field is described by a map $A: \mathbb{M}^{++} \cup \mathbb{M}^{--} \rightarrow H(n)$, which takes the values in the space of $n \times n$ Hermitian matrices. In order to find the link to the Yang-Mills theory on the real Minkowski space, we assume that the expansion

$$
\begin{aligned}
A(x, y) & =A(x)+A_{\alpha}(x) y^{\alpha}+A_{\alpha \beta}(x) y^{\alpha} y^{\beta}+\ldots \\
& =A(x)+A_{\alpha}(x) \frac{p^{\alpha}}{p^{2}} h^{1}+A_{\alpha \beta}(x) \frac{p^{\alpha}}{p^{2}} \frac{p^{\beta}}{p^{2}} h^{2}+\ldots
\end{aligned}
$$


holds. From (4.3) we get $A(x)=0$. So, an infinite sequence $A_{\alpha}(x), A_{\alpha \beta}(x), \ldots$ of the fields defined on $M_{\infty}$ describes the holomorphic gauge field.

The connection form and the curvature form of the metric connection $\nabla$ expressed in a holomorphic frame and canonical coordinates are given by

$$
\begin{gathered}
\theta^{s}=\theta^{s}\left[d x^{\mu}+i h d\left(\frac{p^{\mu}}{p^{2}}\right)\right], \\
\Theta^{s}=\Phi_{\mu \nu}^{s}\left[d x^{\mu} \wedge d x^{\nu}+h^{2} d\left(\frac{p^{\mu}}{p^{2}}\right) \wedge d\left(\frac{p^{\nu}}{p^{2}}\right)\right]+i h \mathscr{R}_{\mu \nu}^{s}\left[d x^{\mu} \wedge d\left(\frac{p^{\nu}}{p^{2}}\right)+d x^{\nu} \wedge d\left(\frac{p^{\mu}}{p^{2}}\right)\right],
\end{gathered}
$$

where $\Phi_{\mu \nu}^{s}:=\frac{1}{2}\left(\Theta_{\bar{\mu} \nu}^{s}-\Theta_{\bar{\nu} \mu}^{s}\right)$ and $\mathscr{R}_{\mu \nu}^{s}:=\frac{1}{2}\left(\Theta_{\bar{\mu} \nu}^{s}+\Theta_{\bar{v} \mu}^{s}\right)$. In the unitary frame, because of (3.11), we have $\Phi_{\mu \nu}^{u}=\frac{1}{2}\left(\Theta_{\bar{\mu} \nu}^{u}-\Theta_{\bar{\mu} \nu}^{u+}\right)$ and $\mathscr{R}_{\mu \nu}^{u}=\frac{1}{2}\left(\Theta_{\bar{\mu} \nu}^{u}+\Theta_{\bar{\mu} \nu}^{u+}\right)$. From the (4.5), (4.6) and from

$$
\begin{gathered}
\theta_{\mu}^{s}(x, p)=-i A_{\mu}(x) h^{0}+\left(\frac{\partial}{\partial x^{\mu}} A_{\alpha}(x)-2 i A_{\mu \alpha}(x)+i\left[A_{\alpha}(x), A_{\mu}(x)\right]\right) \frac{p^{\alpha}}{p^{2}} h^{1}+\ldots \\
\phi_{\mu \nu}^{s}(x, p)=\frac{1}{2}\left[D_{\mu}, D_{\nu}\right] h^{0}+\left(i D_{\mu} A_{\nu \alpha}(x)-i D_{\nu} A_{\mu \alpha}(x)+\frac{1}{2}\left[A_{\alpha}(x),\left[D_{\mu}, D_{\nu}\right]\right]\right) h^{1}+\ldots, \\
f^{-1} \lambda_{\bar{\mu}}(x, p)=\lambda_{\bar{\mu}}(x) h^{0}+\lambda_{\bar{\mu} \alpha}(x) \frac{p^{\alpha}}{p^{2}} h^{1}+\ldots, \\
f^{-1} \Lambda_{\bar{\mu} \bar{\nu}}(x, p) f=\Lambda_{\bar{\mu} \bar{\nu}}(x) h^{0}+\Lambda_{\bar{\mu} \bar{\nu} \alpha}(x) \frac{p^{\alpha}}{p^{2}} h^{1}+\ldots
\end{gathered}
$$

where $D_{\alpha}:=\frac{\partial}{\partial x^{\alpha}}-i A_{\alpha}(x)$, we have

$$
\begin{gathered}
\lim _{y \rightarrow 0} \theta^{s}=-i A_{\alpha}(x) d x^{\alpha}, \\
\lim _{y \rightarrow 0} \Theta^{s}=\frac{1}{2}\left[D_{\alpha}, D_{\beta}\right] d x^{\alpha} \wedge d x^{\beta}=: \frac{1}{2} F_{\alpha \beta}(x) d x^{\alpha} \wedge d x^{\beta} .
\end{gathered}
$$

Therefore, according to (4.11), (4.12) and the expansion (4.4), the Yang-Mills field is a first-order approximation (in parameter $h$ ) of holomorphic gauge field $f=\exp A$.

As it follows from (4.6) $\lim _{h \rightarrow 0} \Theta^{s}$ does not depend on the Hermitian part $\mathscr{R}_{\mu \nu}^{u}$ of curvature form $\Theta_{\bar{\mu} v}^{u}$. Taking this into account, we replace in the Lagrangian (3.15) the term $\operatorname{Tr} \Theta^{u} \wedge * \Theta^{u}$ by $\operatorname{Tr} \Phi^{u} \wedge * \Phi^{u}$, where $\Phi^{u}=\Phi_{\bar{\mu} v}^{u} d \bar{z}^{\mu} \wedge d z^{v}$. The variational principle applied to this new Lagrangian gives the field equations which are similar to (3.19) and (3.20), the only difference being that $\Theta^{s}$ is replaced by $\Phi^{s}$. In the index notation they have the form

$$
\begin{gathered}
\partial^{v} \Phi_{\bar{\mu} \nu}^{s}+\left[\theta^{s v}, \Phi_{\bar{\mu} \nu}^{s}\right]=\frac{1}{2}\left[\psi^{s}\left(\nabla_{\mu} \psi^{s}\right)^{+} H+\psi^{s}\left(f^{-1} \lambda_{\mu}\right)^{+} H+\bar{\partial}^{v} f^{-1} \Lambda_{\bar{\mu} \bar{v}} f\right] \\
\bar{\partial}^{v} \Phi_{\bar{v} \mu}^{s}=\frac{1}{2}\left[\left(\nabla_{\mu} \psi^{s}\right) \psi^{s^{+}} H+f^{-1} \lambda_{\mu} \psi^{s^{+}} H+H^{-1} \bar{\partial}^{v}\left(f^{-1} \Lambda_{\bar{\mu} \bar{v}} f\right)^{+} H\right] \\
\nabla_{\mu} \nabla^{\mu} \psi^{s}+V^{\prime}\left(\psi^{s^{+}} H \psi^{s}\right) \psi^{s}+\nabla_{\mu} f^{-1} \lambda^{\mu}=0 \\
\bar{\partial}^{v}\left(\nabla_{v} \psi^{s}\right)^{+}+\left(\nabla_{v} \psi^{s}\right)^{+} \theta^{+v}+V^{\prime}\left(\psi^{s^{+}} H \psi^{s}\right) \psi^{s^{+}}+\bar{\partial}^{v}\left(f^{-1} \lambda_{v}^{s}\right)^{+}+\left(f^{-1} \lambda_{v}\right)^{+} \theta^{+v}=0
\end{gathered}
$$


Let us now discuss the field equations using the expansion (4.4) and

$$
\psi^{s}(z)=\psi^{s}\left(x^{\alpha}+i h \frac{p^{\alpha}}{p^{2}}\right)=\psi(x) h^{0}+\frac{\partial \psi}{\partial x^{\alpha}}(x) \frac{p^{\alpha}}{p^{2}} h^{1}+\ldots .
$$

After substituting (4.4), (4.11), (4.12), and (4.15) into (4.13) and (4.14) and bringing together the terms of the same order in $h$ we find the infinite sequence of the equations for the fields $A_{\alpha}, A_{\alpha \beta}, \ldots$ and the derivatives $\psi(x), \frac{\partial \psi}{\partial x^{\alpha}}(x), \ldots$ In such a way we get that the equations

$$
\left.\begin{array}{rl}
D^{\nu} F_{v \mu}(x)= & \psi(x)\left(D_{\mu} \psi(x)\right)^{+}-\left(D_{\mu} \psi(x)\right) \psi^{+}(x)+\psi(x) \lambda_{\mu}^{+}(x)-\lambda_{\mu}(x) \psi^{+}(x) \\
& +\frac{\partial}{\partial x_{v}} \frac{1}{2}\left(\Lambda_{\mu v}(x)-\Lambda_{\mu \nu}^{+}(x)\right)-\frac{1}{2 i}\left(\Lambda_{\mu}^{\mu}(x)+\Lambda_{\mu}^{\mu+}(x)\right) \\
D_{\mu} A_{\alpha}^{\alpha}(x) & -D^{\mu} A_{\mu \alpha}(x)=\frac{1}{2}\left[\psi(x)\left(D_{\mu} \psi(x)\right)^{+}+\left(D_{\mu} \psi(x)\right) \psi^{+}(x)\right] \\
& +\psi(x) \lambda_{\mu}^{+}(x)+\lambda_{\mu}(x) \psi^{+}(x)+\frac{\partial}{\partial x_{v}} \frac{1}{2}\left(\Lambda_{\mu v}(x)+\Lambda_{\mu \nu}^{+}(x)\right) \\
& -\frac{1}{2 i}\left(\Lambda_{\mu \alpha}^{\alpha}(x)-\Lambda_{\mu \alpha}^{\alpha+}(x)\right),
\end{array}\right\}
$$

are zero-order approximations of (4.13) and (4.14). Putting $\lambda_{\mu}(x)=0$ and $\Lambda_{\bar{\mu} \bar{\nu}}(x)=0$, we find that (4.16) and (4.17) are equations on the Yang-Mills field $A_{\alpha}(x)$ and on the matter field $\psi(x)$ which hold in standard field theory. If we put $\psi=0, \lambda=0$, and $\Lambda=0$, Eqs. (4.13) could be written in the form

$$
\bar{\partial}^{v} \Phi_{\bar{\mu} \nu}^{s}=0, \quad \partial^{v}\left(H \Phi_{\bar{\mu} v}^{s} H^{-1}\right)=0 .
$$

The perturbative method with respect to $h$ gives the Yang-Mills equations $D^{\mu} F_{\mu \nu}(x)=0$ for $A_{\alpha}(x)$, see Eq. (4.16), and the remaining fields $A_{\alpha \alpha_{1}} \ldots \alpha_{n}(x)$, where $n=1, \ldots$, satisfy

$$
\begin{gathered}
D_{\beta} A_{\alpha \alpha_{2} \ldots \alpha_{n}}^{\alpha}(x)-D^{\alpha} A_{\beta \alpha \alpha_{2} \ldots \alpha_{n}}(x)=j_{\beta \alpha_{2} \ldots \alpha_{n}}(x) \\
D^{\alpha}\left(D_{\beta} A_{\alpha \alpha_{1} \ldots \alpha_{n}}(x)-D_{\alpha} A_{\beta \alpha_{1} \ldots \alpha_{n}}(x)+\left[A_{\alpha_{1} \ldots \alpha_{n}}^{\alpha}(x), F_{\beta \alpha}(x)\right]=k_{\beta \alpha_{1} \ldots \alpha_{n}}(x),\right.
\end{gathered}
$$

where the currents $j_{\beta \alpha_{2} \ldots \alpha_{n}}(x), k_{\beta \alpha_{1} \ldots \alpha_{n}}(x)$ are the algebraic combinations of $A_{\alpha}(x)$, $A_{\alpha \alpha_{1}}(x), \ldots, A_{\alpha \alpha_{1} \ldots \alpha_{n-1}}(x)$ and their derivatives. E.g. for $n=1$ we have $j_{\beta}(x)=0$ and $k_{\beta \alpha_{1}}(x)=0$.

Here arises a problem of consistency of this infinite sequence of the differential equations and their compatibility with the Yang-Mills equations. Extending the finite system of equations for the fields $A_{\alpha}(x), A_{\alpha \alpha_{1}}(x), \ldots, A_{\alpha \alpha_{1} \ldots \alpha_{n-1}}(x)$ by Eq. (4.19) we simultaneously introduce the additional field $A_{\alpha \alpha_{1} \ldots \alpha_{n}}(x)$. Therefore, assuming that the system of differential equations for the fields of the order $\leqq n-1$ 
is consistent, the operation of adding Eq. (4.19) does not destroy the consistency. However, adding Eq. (4.20) to it leads to the following condition

$$
D^{\beta} j_{\beta \alpha_{2} \ldots \alpha_{n}}(x)+k_{\beta \alpha_{2} \ldots \alpha_{n}}^{\beta}(x)=0
$$

on the fields $A_{\alpha}(x), A_{\alpha \alpha_{1}}(x), \ldots, A_{\alpha \alpha_{1} \ldots \alpha_{n-1}}(x)$.

Proposition 3. Condition (4.21) is satisfied when the field $A_{\alpha}(x)$ satisfies the YangMills equations and the fields $A_{\alpha \alpha_{1}}(x), \ldots, A_{\alpha \alpha_{1} \ldots \alpha_{n-1}}(x)$ satisfy (4.19) and (4.20).

Proof. Using (4.19) and (4.20) and the Yang-Mills equations we obtain

$$
\sum_{l=0}^{n-1}\left[\Gamma_{\alpha_{1} \ldots \alpha_{l}}^{v \mu} \Phi_{\mu v \alpha_{l+1} \ldots \alpha_{n-1}}\right]=D^{\beta} j_{\beta \alpha_{1} \ldots \alpha_{n-1}}+k_{\beta \alpha_{1} \ldots \alpha_{n-1}}^{\beta},
$$

where $\Gamma_{\alpha_{1} \ldots \alpha_{k}}^{\nu \mu}$ and $\Phi_{\mu v \alpha_{1} \ldots \alpha_{k}}$ are coefficients appearing in the expansions

$$
\Gamma^{\nu \mu}:=\partial^{v} \theta^{\mu}-\partial^{\mu} \theta^{\nu}-\left[\theta^{\mu}, \theta^{\nu}\right]=\sum_{k=0}^{\infty} \Gamma_{\alpha_{1} \ldots \alpha_{k}}^{\nu^{\mu}} \frac{p^{\alpha_{1}} \ldots p^{\alpha_{k}}}{\left(p^{2}\right)^{k}} h^{k},
$$

and

$$
\Phi_{\mu \nu}=\sum_{k=0}^{\infty} \Phi_{\mu \nu \alpha_{1} \ldots \alpha_{k}} \frac{p^{\alpha_{1}} \ldots p^{\alpha_{k}}}{\left(p^{2}\right)^{k}} h^{k}
$$

respectively. Because equality $\Gamma^{\nu \mu}=0$ holds for $\theta_{\mu}=H^{-1} \partial_{\mu} H$, and because of (4.22) the consistency condition, (4.21) is fulfilled.

We do not know if condition (4.21) is sufficient for the existence of the solutions of (4.19) and (4.20). If it turns out to be sufficient, then by solving the Yang-Mills equations and the infinite sequence of Eqs. (4.19) and (4.20) we could construct the solution of (4.18).

Conversely, let $f$ be the solution of (4.18). Then, $f^{\prime}=g f$, where $g=f\left(z^{\mu}, z^{\mu}\right)^{-1}$ [in $f\left(z^{\mu}, \bar{z}^{\mu}\right)$ we have replaced $z^{\mu}$ by $z^{\mu}$ ] is also solution of (4.18), which is a consequence of gauge invariance of (4.18). Besides that we have $\lim _{y \rightarrow 0} f^{\prime}=$ id, i.e. the condition (4.3) is satisfied. Thus, considering the correspondence of (4.18) to $D^{\mu} F_{\mu \nu}(x)=0$ and (4.11) we find that

$$
-i A_{\mu}^{\prime}(x)=\lim _{y \rightarrow 0}\left[\left(f^{-1} \bar{\partial}_{\mu} f\right)^{+}-f^{-1} \bar{\partial}_{\mu} f\right]
$$

is a solution of the Yang-Mills equations.

It is important to note, in context of considerations presented at the end of Sect. 2 (where we put $h=$ Planck constant), that the significant difference between holomorphic and standard field theory appears in high energy limit.

Acknowledgements. I am thankful to Prof. K. Maurin for his interest and encouragement which helped me to do this research. I want also to thank Prof. L. S. Woronowicz for a critical reading of manuscript and stimulating discussions. 


\section{References}

1. Abraham, R., Marsden, J.E.: Foundation of mechanics 2nd ed. Reading, MA: Benjamin 1978

2. Atiyah, M.F., Hitchin, N.J., Singer, I.M.: Self-duality in four dimensional Riemannian geometry. Proc. R. Soc. Lond. A 362, 425-461 (1978)

3. Gawedzki, K.: Fourier-like kernels in geometric quantization. Dissertationes Mathematicae, p. 128 (1976)

4. Griffiths, P., Harris, J.: Principles of algebraic geometry. New York: Wiley 1978

5. Guillemin, V., Sternberg, S.: Geometric asymptoties. Providence, RI: Am. Math. Soc. 1977

6. Jacobsen, H.P., Vergne, M.: Wave and Dirac operator, and representations of the conformal group (preprint)

7. Karpio, A., Kryszeń, A., Odzijewicz, A.: Two-twistor conformal hamiltonian spaces. Will appear in Rep. Math. Phys.

8. Manin, Y.I.: Kalibrovochnye pola i holomorfnaja geometria. Sowremiennye problemy matematyki, Vol. 17, pp. 3-55. M.: Viniti 1981

9. Odzijewicz, A.: A holomorphic field theory. Lett. Math. Phys. 8, 329-335 (1984)

10. Odzijewicz, A.: Conformal kinematics and twistor flag spaces. Teubner-Texte zur Mathematik 34, 47-55 (1981)

11. Penrose, R., MacCallum, M.A.H.: Twistor theory: An approach to the quantisation of fields and space-time. Phys. Rep. C6, 241-316 (1972)

12. Penrose, R.: The twistor programme. Rep. Math. Phys. 12, 65-76 (1977)

13. Rühl, W.: On conformal invariance of interacting fields. Commun. Math. Phys. 34, 149-166 (1973)

14. Wells, P.O., Jr.: Hyperfunction solutions of the zero-restmass fields equations. Commun. Math. Phys. 78, 567-600 (1981)

Communicated by R. Haag

Received February 20, 1986 
